\title{
Impacto de los Métodos Aplicados para la Determinación de la Permeabilidad y su Influencia en el Gerenciamiento del Reservorio en el Campo Shushufindi
}

\author{
Guamialamá, Carlos $^{1 *}$ iD ; Freire, José ${ }^{2}$ iD; Valencia, Raúl ${ }^{1}$ i \\ ${ }^{1}$ Escuela Politécnica Nacional, Facultad de Ingeniería en Petróleos, Quito, Ecuador \\ ${ }^{2}$ Schlumberger del Ecuador S.A., Production Management, Quito, Ecuador
}

\begin{abstract}
Resumen: Permeabilidad es una de las principales propiedades en la caracterización de yacimientos, así como para la estimación de la productividad de un pozo. Existen diversos métodos para su determinación durante diferentes etapas de desarrollo de un campo. Todos los métodos difieren en algunos aspectos como el tipo de permeabilidad que determinan ya sea absoluta o efectiva, radio de investigación, tecnología utilizada, fundamentos teóricos y desventajas encontradas durante la adquisición y estimación de esta propiedad. En este trabajo se determinó las distribuciones de permeabilidad y se comparó entre los métodos núcleos-registros eléctricos, registros eléctricos-probadores de formación y pruebas de presión-análisis transiente de producción. A partir de estas comparaciones, se identificaron las diferencias en los valores de permeabilidad así como las diferencias en las escalas de medición. Una vez establecidos los valores de permeabilidad a partir de cada método, se realizó un análisis e interpretación, determinando una productividad teórica de cada uno de ellos, y comparándola con datos de producción real se estableció el mejor método para la estimación de esta propiedad para el campo Shushufindi.
\end{abstract}

Palabras clave: Permeabilidad, núcleos, registros, productividad, pruebas de presión.

\section{Impact of the Applied Methods for the Determination of Permeability and its Influence in the Management of the Reservoir in Shushufindi Field}

\begin{abstract}
Permeability is one of the main properties in reservoir characterization, as well as for the estimation of the productivity of a well. There are several methods for its measurement or calculation during different stages of development of a field. All these methods differ in some aspects such as the type of permeability that they determine whether absolute or effective, volume of observation, technology used, theoretical foundations and inconveniences encountered during the acquisition and estimation of this property. In this work, permeability distributions were determined, and the methods were compared with core-well logs, well logs-wireline formation testers and pressure transient analysis - rate transient analysis. From these comparisons, it was possible to identify the differences in the permeability values as well as the differences in the measurement scales. Once the permeability values were established from each method, an analysis and interpretation were made to determine a theoretical productivity of each of them, then they were compared with real production data in order to establish the best method for estimating this property for Shushufindi field.
\end{abstract}

Keywords: Permeability, core, well logs, productivity, well test.

\section{INTRODUCCIÓN}

El campo Shushufindi está ubicado en la Cuenca Oriente del Ecuador (López et al, 2015), fue descubierto en 1969 con la perforación del pozo Shushufindi 1. La producción del campo arrancó en agosto de 1972 alcanzando su pico en agosto de 1986 con un promedio diario para ese mes de 126400 barriles. Shushufindi es considerado uno de los campos gigantes del
Ecuador y la perfección de su sistema le ha permitido entregar a Ecuador la mayor riqueza natural, sobrepasando en los primeros meses del año 2002 los 1000 millones de barriles de producción acumulada de alrededor de 42 años. Para el año 2013, sobrepasaba ya los 1200 millones de barriles provenientes en orden de importancia del reservorio $\mathrm{T}(65 \%)$, U (35\%) y Tena Basal (<1\%). El Campo Shushufindi presenta una secuencia sedimentaria y volcánica, de edades del

*carlos.guamialama@epn.edu.ec

Recibido: 21/08/2019

Aceptado: 29/04/2020

Publicado: 31/05/2020

$10.33333 /$ rp.vol45n2.04

CC BY 4.0 
Paleozoico al Cuaternario, con una profundidad que oscila entre los 8.000 a 12.000 [ft], que descansan sobre un substrato precámbrico. Las principales rocas reservorio, involucran facies de la formación Napo de edad Cretácico Inferior y areniscas zona U y T. La composición de estas areniscas es principalmente cuarzosa de grano fino a medio, de regular a buena selección. El cemento es silíceo o calcáreo y la matriz arcillosa, con presencia de glauconita en los miembros superiores (Baby et al, 2014).

Históricamente este campo no ha dado mayores problemas en su operación gracias a la nobleza de los reservorios y a la perfección de sus mecanismos de empuje, sin embargo, en la actualidad plantea un reto mayor, determinar cuál es el régimen óptimo de producción y recuperación mejorada para su etapa de madurez, lo cual constituye a la permeabilidad como una de las propiedades más importantes en la determinación del potencial de producción de un pozo, caracterización del reservorio, así como para la simulación de modelos dinámicos.

La permeabilidad ha llegado a ser percibida por la industria como una de las propiedades más desafiantes de determinar. Las dificultades pueden ser atribuidas fundamentalmente a su naturaleza, la cual es una función de la dirección, volumen de investigación, así como de las interacciones roca-fluido y fluido-fluido. La principal influencia de la permeabilidad se evidencia en el movimiento de fluidos, además es uno de los datos de entrada utilizados para los modelos de simulación usados en la predicción de producción.

Cuanto más real sean los modelos de simulación, esto permitirá la integración de los datos de permeabilidad obtenidos a partir de diferentes métodos, el grado de confiabilidad puede ser situado en la habilidad para predecir la producción de un pozo, así como el comportamiento de un reservorio.

Ninguno de los métodos disponibles para determinar la permeabilidad, sean estos análisis de núcleos, registros eléctricos, probadores de formación con cable, pruebas de pozos, y análisis transiente de producción, pueden trabajar individualmente (Al-Harbi et al, 2007).

\section{MARCO TEÓRICO}

\subsection{Definición de permeabilidad}

Permeabilidad es la medida de la habilidad que tiene una roca para transmitir fluidos, medida típicamente en milidarcies, la cual es la milésima parte de un Darcy. Un Darcy describe la permeabilidad de un medio poroso a través del cual se produce el paso de un centímetro cúbico de fluido que tiene un centipoise de viscosidad y fluye en un segundo bajo una presión diferencial de una atmósfera, donde el medio poroso expone un área en sección transversal de un centímetro cuadrado y una longitud de un centímetro (Schlumberger, 2019).

Se han definido tres conceptos de permeabilidad: absoluta, propiedad intrínseca de la roca cuando está saturada al cien por ciento por un fluido de una sola fase; efectiva, medida en referencia a un fluido cuando la roca está saturada por un fluido multifásico, y relativa, relación entre la permeabilidad efectiva y absoluta (Sullivan et al, 2008).

Para estimar la permeabilidad, lo podemos hacer a diferentes escalas; mediante pruebas de laboratorio efectuadas en núcleos. Se la infiere a gran escala a través de pruebas de pozos y datos de producción. También se la cuantifica a una escala intermedia mediante los probadores de formación operados con cable (Schlumbeger, 2002).

\subsection{Núcleos}

Los datos de permeabilidad de núcleos son obtenidos de varios pozos, a medida que se perfora la zona de interés, estas constituyen una pequeña muestra en comparación al tamaño del yacimiento, es una manera directa de medir la permeabilidad de un medio poroso, esto independientemente del volumen de la muestra en relación al yacimiento en análisis (Al-Harbi et al, 2007).

Los análisis de rutina de núcleos proveen la permeabilidad absoluta o intrínseca, tanto horizontal como vertical, mientras que un análisis especial determina propiedades como las permeabilidades relativas (Schlumberger, 2002).

La permeabilidad puede determinarse en laboratorio midiendo la cantidad de fluido que pasa a través de una muestra cuando se aplica un diferencial de presión a lo largo de la misma, los instrumentos utilizados para este fin se denominan permeámetros. Los permeámetros cuentan con una cámara en la cual se ubica una muestra, usualmente un tapón de núcleo (que puede ser horizontal o vertical). La permeabilidad es medida haciendo pasar un fluido de viscosidad $\mu$ conocida a través de núcleos, al cual se le han medido las dimensiones (A y L). Luego se determina la tasa de flujo q y la caída de presión $\Delta \mathrm{P}$; una vez obtenidos todos estos parámetros la permeabilidad es calculada con la ecuación 1 conocida como la ecuación de Darcy:

$$
k=\frac{q \mu L}{A \Delta P}
$$

Donde:

$\mathrm{K}=$ permeabilidad absoluta

$\mathrm{q}=$ caudal

$\mu=$ viscosidad

$\mathrm{L}=$ longitud del núcleo

$\mathrm{A}=$ área transversal del núcleo

$\Delta \mathrm{P}=$ diferencial de presión

Un aspecto importante a considerar son las anormalidades en los datos, esto debido a inconvenientes que ocurren durante el corte del núcleo en el laboratorio, la mayor parte de la muestra es invadida por el agua utilizada para su corte, la presencia de agua y la cortadora causan ciertos daños en las caras de los núcleos. El alisado del tope y la base del núcleo utilizando la cortadora causa reposicionamiento de los granos, estos granos causan la reducción de la permeabilidad, por otra parte si el agua utilizada para el corte del núcleo es incompatible con la estabilidad de las arcillas del núcleo, estas arcillas pueden hincharse causando cambios en la permeabilidad (Talabani et al, 1999). 


\subsubsection{Relación porosidad permeabilidad}

El predictor de la permeabilidad más usado es la relación de Porosidad- Permeabilidad. Es reconocido que la mayoría de las rocas de los yacimientos presenta una porosidad primaria, la misma que en una escala semilogarítmica muestra una relación razonablemente lineal entre estas dos propiedades. Una buena práctica en la estimación de la permeabilidad consiste en la separación en grupos o categorías que muestren un comportamiento más homogéneo (Castillo y Ortega, 2016).

\subsubsection{Efecto Klinkenberg}

Las mediciones de permeabilidad se restringen a regiones de bajas tasas de flujo (flujo laminar). En casos de altas tasas de flujo, la ecuación de Darcy no es adecuada para describir la relación entre la tasa de flujo y la caída de presión (Torbello, 2012).

\subsection{Registros eléctricos}

Los registros se han convertido en una herramienta fundamental para la evaluación de formaciones, son corridos una vez culminada la etapa de perforación de un pozo. Los registros se basan en la medición de propiedades físicas, con una amplia variedad de herramientas, no existe un registro geofísico específico para la determinación directa de la permeabilidad, pero es posible su determinación mediante el análisis de registros convencionales (Shokir et al, 2006).

\subsubsection{Métodos para la estimación de la permeabilidad a través de registros}

El cálculo de la permeabilidad a partir de registros de pozos tiene varios métodos aplicables como métodos empíricos, métodos estadísticos, y medidas virtuales, haciendo uso de ecuaciones empíricas, múltiples regresiones variables y redes neuronales artificiales respectivamente (Mohaghegh et al, 1997).

La estimación de la permeabilidad con métodos empíricos se realiza mediante la aplicación de una variedad de correlaciones de varios autores como Coates, Tixier, Salazar, y Timur, todas estas correlaciones pueden ser simplificadas con la ecuación 2 (Bazara y Salman, 2009):

$$
\mathrm{k}=\left(\frac{\mathrm{a} \emptyset^{\mathrm{b}}}{\mathrm{Swic}}\right)^{\mathrm{d}}
$$

Donde:

$\mathrm{K}=$ permeabilidad absoluta $[\mathrm{mD}]$

$\emptyset=$ porosidad

Swi $=$ saturación de agua irreductible

$\mathrm{a}, \mathrm{b}, \mathrm{c}, \mathrm{d}=$ coeficiente $\mathrm{y}$ exponentes detallados en la tabla 1 .
Tabla 1. Valores de coeficientes y exponentes métodos empíricos

\begin{tabular}{lcccc} 
Método & Coeficientes & \multicolumn{3}{c}{ Exponentes } \\
& $\mathrm{a}$ & $\mathrm{b}$ & $\mathrm{c}$ & $\mathrm{d}$ \\
\hline Coates & 100 & 2 & 1 & 2 \\
Tixier & 250 & 3 & 1 & 2 \\
Salazar & $4,22 \times 10^{6}$ & 7,5 & 0,09 & 1 \\
Timur & 0,136 & 4,4 & 2 & 1 \\
\hline
\end{tabular}

Las múltiples regresiones variables son un método estadístico, el cual busca una relación entre la permeabilidad de los núcleos con otros parámetros de los registros.

Las redes neuronales son una inteligencia artificial que se basa en un proceso secuencial y algorítmico, utiliza una amplia gama de procesos simples en lugar de utilizar un solo proceso complejo. Este consiste en un entrenamiento que se va propagando en la red neuronal, durante este entrenamiento se le proporciona problemas previamente resueltos que incrementan el conocimiento de la red, una vez finalizado este entrenamiento la red neuronal sea capaz de procesar datos de registros y dar como resultado el cálculo de la permeabilidad (Mohaghegh et al, 1997).

Los registros tienen un radio de investigación muy limitado como se muestra en la Figura 1, debido a la resolución de las herramientas empleadas para la toma de datos, además son influenciados por el factor de laminación y el filtrado de la zona invadida, pero con el principal beneficio de ser una medida continua (Cantini et al, 2013).

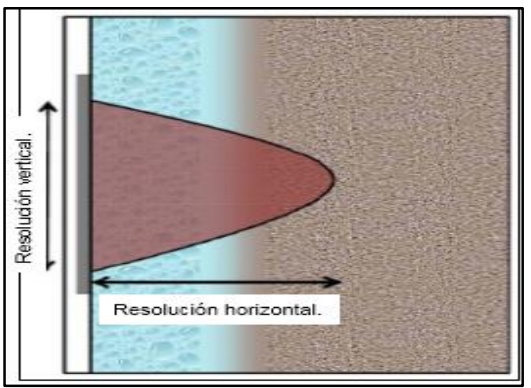

Figura 1. Radio de investigación herramientas de registros

\subsection{Probadores de formación con cable}

Los probadores de formación con cable (xpt) Figura 2, son utilizados principalmente en pozos recién perforados durante la corrida de registros convencionales para obtener parámetros de los intervalos de interés como: presión de la formación, movilidad, así como para recuperar muestras de fluidos de la formación. Previo a la toma de presiones puntuales con los probadores de formación se debe seleccionar los puntos con el apoyo de registros (aún sin interpretar) SP, GR y resistivos a fin de encontrar la zona de interés a evaluar. Este tipo de herramientas cuenta con un bloque que contiene un empaque sellador, opuesto a un brazo que abre a la profundidad de interés, forzando al bloque y al empaque sellador para que entren en contacto firme con la formación (Pérez, 2012). 


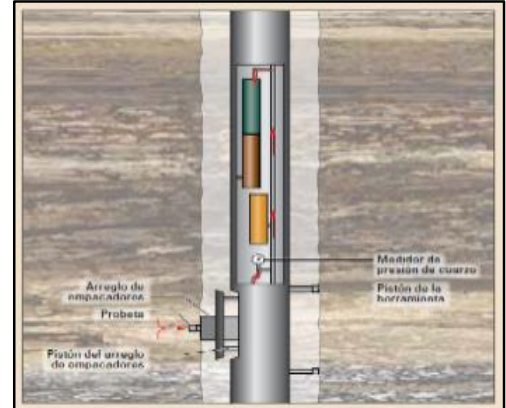

Figura 2. Configuración probador de formación con cable

La evaluación con la herramienta empieza con la extracción de un pequeño volumen de fluido, típicamente entre 5 y 25 centímetros cúbicos desde la formación hacia la cámara de la herramienta. La primera etapa es llamada drawdown la cual es una caída de presión que depende principalmente de la permeabilidad de la zona evaluada, la viscosidad del fluido, y la tasa de fluido que ingresa a la cámara. Si el punto evaluado tiene la suficiente permeabilidad se lleva a cabo una segunda etapa la cual toma datos de incremento de presión.

Los probadores de formación con cable ayudan al cálculo de la permeabilidad a partir de los datos drawdown o build up, la permeabilidad calculada es efectiva, principalmente esférica y ocasionalmente horizontal (Cantini et al, 2013).

Debido a que el disturbio de presión ocasionado por un probador de formación con cable tiene un radio de investigación muy corto, normalmente dicho disturbio se da dentro de la región donde es más probable que ocurra invasión de filtrado de lodo ya que la mayor parte de estas pruebas se realiza en pozos recién perforados (Schlumberger, 1996). Por lo tanto, la movilidad determinada con el análisis de los decrementos e incrementos de presión permite calcular la permeabilidad efectiva al filtrado de lodo en la zona de invasión. Sin embargo, se puede considerar como indicador cualitativo de productividad.

\subsection{Análisis transiente de producción}

Es una técnica relativamente nueva que permite determinar las características del yacimiento, sustentada en métodos empíricos básicos como Arps, Fetkovich, hasta el actual y moderno Blasingame. El análisis de estas curvas facilita la obtención de las propiedades como la permeabilidad efectiva del reservorio sin detener la producción de un pozo. Este método recopila datos diarios de pruebas de tasas de flujo y presión.

Las curvas de declinación de producción tienen dos funciones principales, la primera de ellas es la de determinar la permeabilidad efectiva mediante el empate de la curva de declinación de producción con las curvas tipo, otra función es en base a un diagnóstico, realizar una predicción de la producción (Sun, 2015).

En la actualidad uno de los métodos tradicionales consiste en la aplicación de las curvas tipo de Blasingame Figura 3.

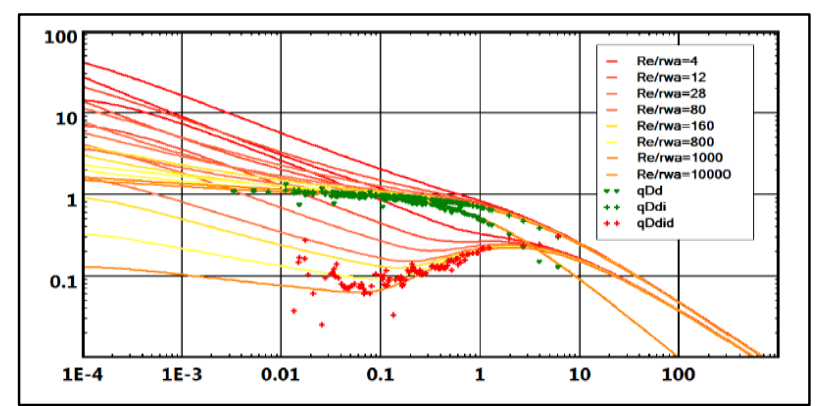

Figura 3. Curvas análisis transiente de producción

Para la determinación de la permeabilidad, se debe realizar el match point con la tasa de producción, una vez obtenido este punto, se aplica la ecuación 3 para estimar la permeabilidad:

$$
k=\frac{\left(\frac{q}{\Delta p_{p}}\right)_{M} \mu \beta}{\left(q_{D d}\right)_{M} 2 \pi h}\left(l n r_{e D}-\frac{1}{2}\right)
$$

Donde:

$\mathrm{K}=$ permeabilidad efectiva $[\mathrm{mD}]$

$\left(\frac{q}{\Delta p_{p}}\right)_{M}=$ match point tasa de producción

$\mu=$ viscosidad $[\mathrm{cp}]$

$\beta$ = factor volumétrico petróleo [BF/BY]

$q_{D d}=$ tasa de producción [BPPD]

$\mathrm{h}=$ espesor de la arena [ft]

$\mathrm{r}_{\mathrm{ed}}=$ radio de drenaje del pozo [ft]

\subsubsection{Relación análisis transiente de producción y presión}

Teóricamente, el análisis de los datos de producción transiente (RTA) es el mismo que se realiza en el análisis transiente de presión (PTA). Los métodos RTA y PTA comparten las mismas asunciones en términos de uso de la ecuación de la difusividad y condiciones de límite. La mayor parte de los modelos desarrollados para análisis PTA puede ser utilizada en el análisis RTA. 
Tabla 2. Comparación PTA-RTA

\begin{tabular}{|c|c|c|}
\hline & $\begin{array}{c}\text { Análisis transiente de } \\
\text { presión. }\end{array}$ & $\begin{array}{c}\text { Análisis } \\
\text { transiente de } \\
\text { producción. }\end{array}$ \\
\hline Bases Teóricas. & \multicolumn{2}{|c|}{$\begin{array}{l}\text { Mismas ecuaciones, superposición, y los } \\
\text { mismos modelos numéricos y analíticos. }\end{array}$} \\
\hline $\begin{array}{l}\text { Rango de } \\
\text { tiempo. }\end{array}$ & Horas, días. & $\begin{array}{l}\text { Semanas, meses, } \\
\text { años. }\end{array}$ \\
\hline $\begin{array}{l}\text { Periodos de } \\
\text { interés. }\end{array}$ & $\begin{array}{l}\text { Principalmente en cierre } \\
\text { de pozos. }\end{array}$ & $\begin{array}{l}\text { Fases de } \\
\text { producción. }\end{array}$ \\
\hline $\begin{array}{l}\text { Datos } \\
\text { requeridos. }\end{array}$ & $\begin{array}{l}\text { Medidas de pruebas de } \\
\text { pozos. } \\
\text { Pruebas de formación. }\end{array}$ & $\begin{array}{l}\text { Datos de } \\
\text { producción. } \\
\text { Presión en } \\
\text { superficie. }\end{array}$ \\
\hline $\begin{array}{l}\text { Área de interés } \\
\text { del yacimiento. }\end{array}$ & $\begin{array}{c}\text { Cualquier volumen e } \\
\text { investigación alcanzada } \\
\text { durante la prueba. }\end{array}$ & $\begin{array}{l}\text { Área de drenaje } \\
\text { del pozo. }\end{array}$ \\
\hline $\begin{array}{l}\text { Diagnósticos } \\
\text { modernos. }\end{array}$ & $\begin{array}{l}\text { Log-Log derivada de } \\
\text { Bourdet. }\end{array}$ & $\begin{array}{l}\text { Log-Log y } \\
\text { Blasingame }\end{array}$ \\
\hline $\begin{array}{l}\text { Régimen de flujo } \\
\text { de interés. }\end{array}$ & Flujo radial infinito. & $\begin{array}{l}\text { Estado pseudo } \\
\text { estacionario. }\end{array}$ \\
\hline $\begin{array}{l}\text { Resultados } \\
\text { principales. }\end{array}$ & Kh y daño. & Kh y daño. \\
\hline $\begin{array}{l}\text { Capacidad de } \\
\text { diagnóstico. }\end{array}$ & Alta a muy alta & Baja en promedio. \\
\hline
\end{tabular}

\subsection{Pruebas de presión}

Las pruebas de presión son una herramienta que se desarrolla a partir de la necesidad de conocer la dinámica del movimiento de los fluidos dentro del reservorio. A través del tiempo, fueron evolucionando, y adquiriendo técnicas que permitieron dar respuestas simples a problemas complejos.

La permeabilidad determinada de las pruebas de presión corresponde a la permeabilidad efectiva; se la calcula usando el análisis transiente de presión moderno, basado en la derivada de la presión versus el tiempo. Esta permeabilidad representa un promedio de esta propiedad en el área de drenaje, con una escala significativamente mayor que las obtenidas de núcleos o registros. Sin embargo, los valores calculados a partir de estas pruebas no son medidas directas de permeabilidad como las medidas tomadas de los núcleos. El dato estimado de estas pruebas, es el promedio de la capacidad de flujo del reservorio, más no el cálculo directo de permeabilidad (Sullivan et al, 2008):

$$
k h=141,2 q \beta \mu\left(\frac{p_{D}}{\Delta p}\right)_{P C}
$$

Donde:

$\mathrm{K}=$ permeabilidad efectiva $[\mathrm{mD}]$

$\mathrm{h}=$ espesor de la arena [ft]

$\mathrm{q}=$ caudal de prueba $[\mathrm{BPPD}]$

$\beta$ = factor volumétrico petróleo [BF/BY]

$\mu=$ viscosidad $[\mathrm{cp}]$

$\left(\frac{p_{D}}{\Delta p}\right)_{P C}=$ match point derivada de la presión
La permeabilidad es calculada a partir de la ecuación 4 conociendo el dato de espesor de la arena que se encuentra en el área de influencia de la prueba.

Si se tiene una variación anisotrópica a gran escala en el reservorio, la estimación de la permeabilidad por medio de pruebas de pozos, será un promedio geométrico de la permeabilidad como lo indica la ecuación 5.

$$
k_{G}=\sqrt{k_{\min } * k_{\max }}
$$

Donde:

$\mathrm{K}=$ promedio geométrico permeabilidad efectiva [mD]

$k_{\text {min }}=$ permeabilidad mínima del volumen de investigación

$k_{\max }=$ permeabilidad mínima del volumen de investigación

Las pruebas de presión convencionales permiten detectar el grado de heterogeneidad, pero no se puede determinar la distribución de la permeabilidad y adicionalmente esta fuente carece de resolución vertical (Schlumberger, 2002).

Los problemas más comunes durante la interpretación de la derivada de datos build up son:

Efecto de almacenamiento el cual ocurre especialmente cuando el cierre del pozo se lo realiza desde superficie, ya que no se tiene un control inmediato del flujo; es decir, el cambio de la tasa al frente de la arena cambia más lento que la tasa en superficie.

Efecto de segregación de fases la cual ocasiona una distorsión de la derivada de la presión.

Efecto de ruido dificulta reconocimiento de los regímenes de flujo, volviéndolo complejo, lo que lleva a la elección errónea del modelo para la interpretación.

\subsection{Comportamiento de afluencia de formaciones productoras}

La determinación de las curvas IPR para pozos de petróleo es extremadamente importante en el análisis de sistemas de producción. Estas curvas son conocidas como la representación gráfica de las presiones fluyentes (Pwf) con la cual el yacimiento entrega en el fondo del pozo distintas tasas de producción (Q). Es decir, para cada Pwf existe una tasa de producción de líquido (Nind, 2010).

Cuando se dispone de la suficiente información se puede hacer uso de la ecuación 6 para la elaboración de las curvas:

$$
J=\frac{0,00708 K_{o} h}{\mu_{o} \beta_{o}\left[\operatorname{Ln}\left(\begin{array}{l}
r_{e} \\
r_{w}
\end{array}\right)-0.75+s\right]}
$$

Donde:

$\mathrm{J}=$ índice de productividad

$\mathrm{K}=$ permeabilidad $[\mathrm{mD}]$

$\mathrm{h}=$ espesor de la arena [ft]

$\mu_{o}=\operatorname{viscosidad}[\mathrm{cp}]$

$\beta_{o}=$ factor volumétrico petróleo $[\mathrm{BF} / \mathrm{BY}]$

$\mathrm{r}_{\mathrm{e}}=$ radio de drenaje del pozo $[\mathrm{ft}]$

$\mathrm{r}_{\mathrm{w}}=$ radio del pozo $[\mathrm{ft}]$

$\mathrm{s}=$ daño del pozo 


\subsection{Variogramas}

La distribución espacial de las propiedades de la roca es un aspecto fundamental en el proceso de caracterización del reservorio. La geoestadística es una rama de las "estadísticas aplicadas" que intenta describir la distribución de una propiedad en el espacio, también se la conoce como estadísticas espaciales. Se supone que una propiedad distribuida espacialmente exhibe algún grado de continuidad. La porosidad y la permeabilidad son ejemplos de espacialidad. El modelado geoestadístico se refiere al procedimiento para determinar un conjunto de realizaciones del reservorio. Las realizaciones dependen tanto de las relaciones espaciales entre los puntos de datos como de su correlación estadística en función del espacio de separación.

Las relaciones espaciales asociadas con los datos se calculan y luego se modelan. Los puntos trazados forman el semivariograma experimental, y la línea que se ajusta a los puntos de datos se denomina modelo de semivariograma, la Figura 4 ilustra un ajuste a los datos por un modelo de semivariograma (Fanchi, 2018).

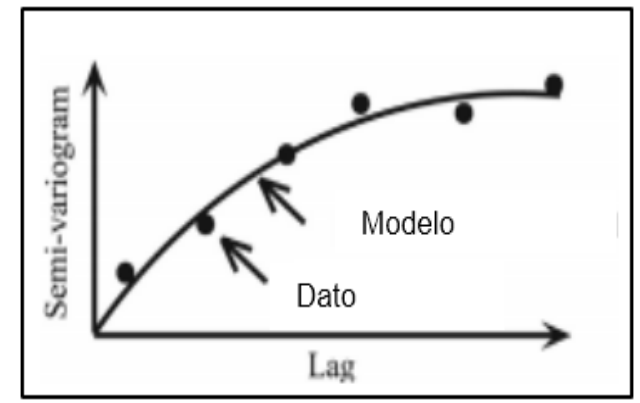

Figura 4. Variograma experimental

Un semivariograma es una gráfica de semivarianza versus rango. La función semivarianza de la ecuación 7 es una medida del grado de disimilitud entre los valores de un parámetro $\mathrm{Z}$ en dos ubicaciones diferentes, o puntos en el espacio. La semivarianza $\gamma(\mathrm{h})$ es una función del intervalo h, o la distancia de separación, entre dos observaciones $\mathrm{Z}(\mathrm{x})$ y $\mathrm{Z}(\mathrm{x}+\mathrm{h})$ del parámetro Z, y se tiene:

$$
\gamma(h)=\frac{1}{2 N(h)} \sum_{i=1}^{N(h)}\left[Z\left(x_{1}\right)-Z\left(x_{2}+h\right)\right]^{\wedge} 2
$$

Donde N (h) es el número de pares que están separados a la distancia $h$.

El estimador así definido lleva el nombre de variograma experimental. No se trata de una función propiamente tal, sino de una serie de valores, pues sólo se puede calcular para vectores h tales que $\mathrm{N}$ (h) no es vacío (Emery, 2013).

\section{RESULTADOS Y DISCUSIÓN}

\subsection{Permeabilidad de núcleos}

\subsubsection{Efecto klinkenberg}

Un aspecto importante a considerar es el efecto klinkenberg Figura 5, la cual representa un grupo de datos de núcleos del pozo SSF-151 donde la permeabilidad absoluta fue medida utilizando aire, con su respectiva corrección en laboratorio por el efecto Klinkenberg.

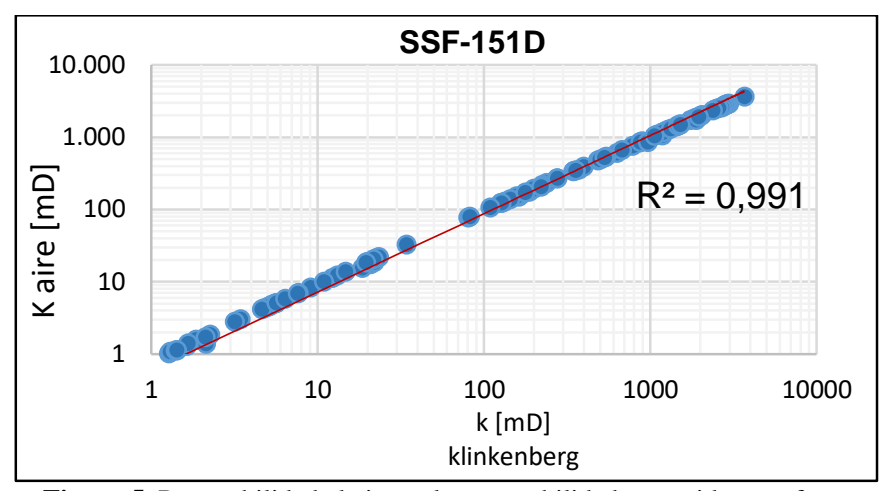

Figura 5. Permeabilidad al aire vs la permeabilidad corregida por efecto klinkenberg

Es importante notar que los valores de las permeabilidades medidas al aire son muy similares a las permeabilidades corregidas por el efecto Klinkenberg, especialmente a valores de permeabilidad mayores a los $100[\mathrm{mD}]$ donde se tiene un mejor ajuste a la línea de tendencia con un coeficiente de determinación $\left(\mathrm{R}^{2}\right)$ de 0.991 .

\subsubsection{Análisis de permeabilidades de núcleos}

La Figura 6 corresponde a la relación permeabilidad-porosidad de 4 pozos del campo Shushufindi de la arenisca U. Esta relación permite identificar tres tipos de roca, donde cada tipo de roca muestra una relación debido a su comportamiento homogéneo. Esta relación porosidad-permeabilidad es fácil de evidenciar en unidades de roca que fueron depositadas bajo las mismas condiciones.

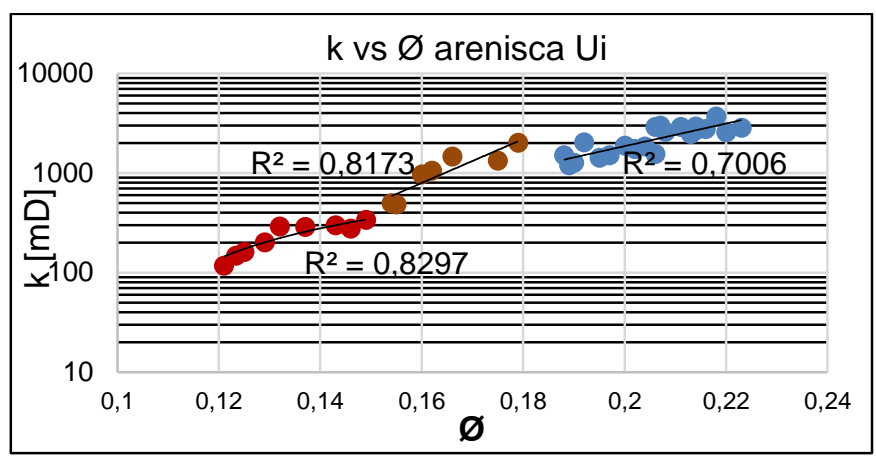

Figura 6. Relación permeabilidad porosidad para la arenisca Ui del campo Shushufindi

Los valores de permeabilidades registradas en cada tipo de roca incrementan directamente con la porosidad. Los coeficiente de determinación $\left(\mathrm{R}^{2}\right)$ mustran una fuerte relación entre las dos propiedades con valores entre $0.7-0.83$

\subsection{Permeabilidades de registros eléctricos}

La Figura 7 corresponde al registro eléctrico del pozo SSF151D donde en la última pista se tiene la permeabilidad calculada con la ecuación empírica de Coates y la permeabilidad de núcleos para la arenisca $\mathrm{U}$ inferior. Si bien el ajuste entre las permeabilidades de núcleos y registros evidencia una diferencia, esta es de esperarse debido a que el 
volumen de investigación de los registros es típicamente 2 o 3 veces mayor que los núcleos.

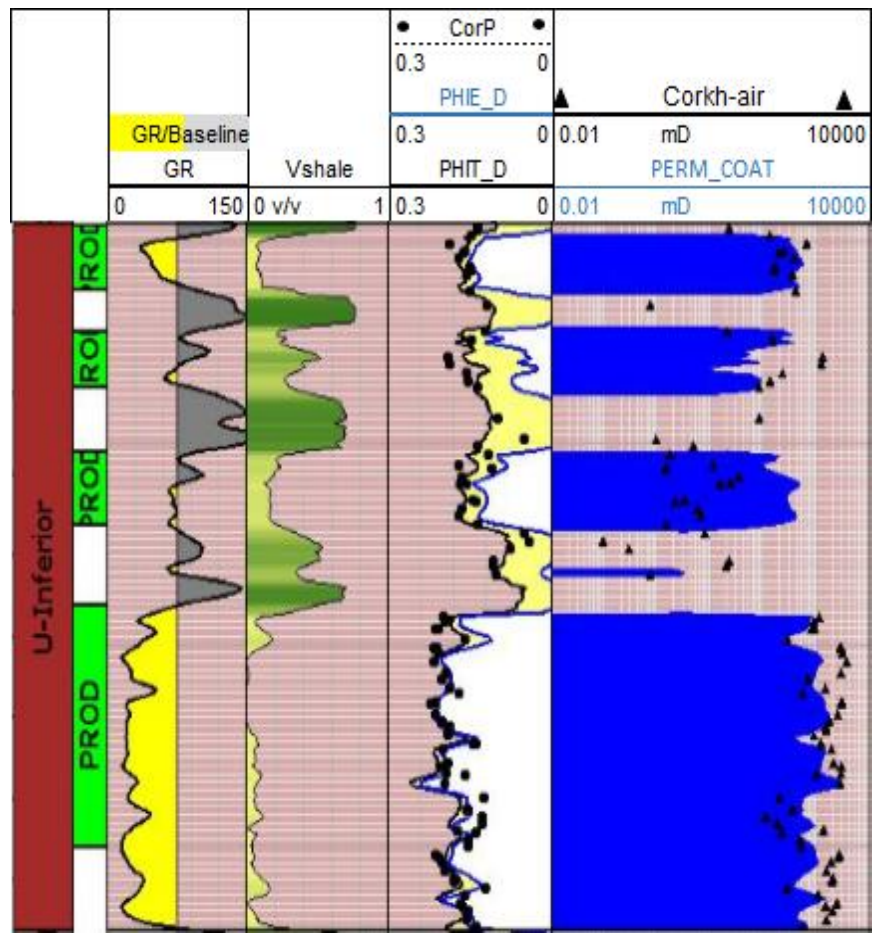

Figura 7. Permeabilidad de Coates y núcleos del pozo Shushufindi-151D de la arenisca $U$ inferior

El reto en el cálculo de la permeabilidad a partir de registros eléctricos radica en la selección de la ecuación empírica apropiada, por lo que los datos de núcleos se vuelven imprescindibles en la calibración de esta fuente para la obtención de la permeabilidad.

\subsection{Permeabilidades de pruebas de pozos}

Las Figuras 8 y 9 muestran los histogramas con la distribución de permeabilidad obtenidos con pruebas de restauración de presión para las areniscas $\mathrm{U}$ y $\mathrm{T}$ inferior del campo Shushufindi.

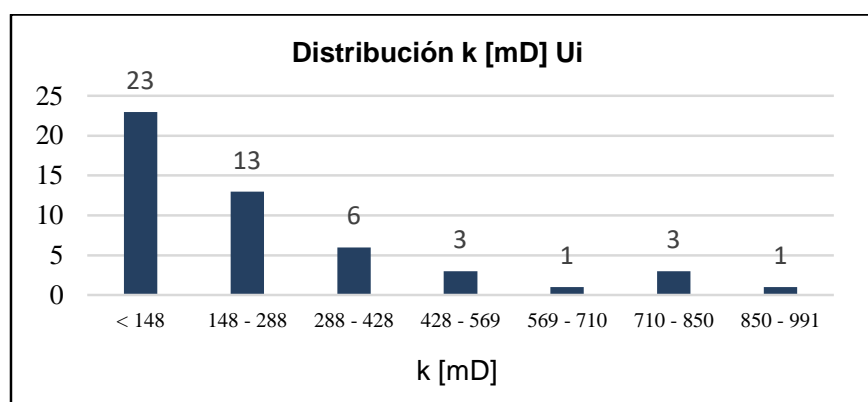

Figura 8. Distribución de permeabilidad efectiva para la arenisca U inferior

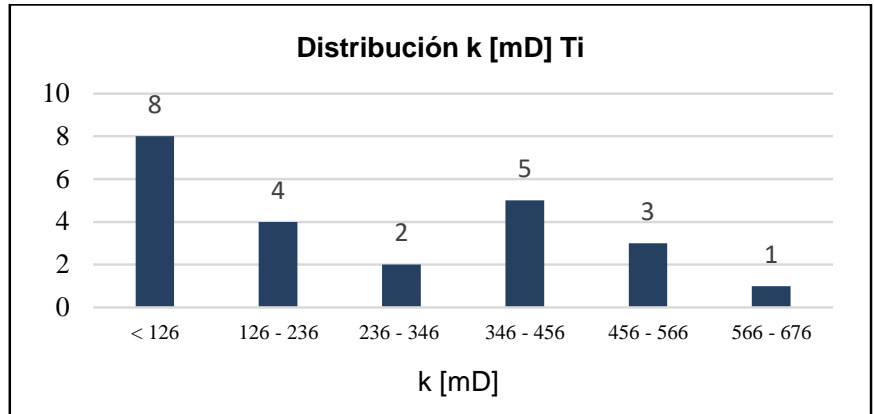

Figura 9. Distribución de permeabilidad efectiva para la arenisca T inferior

La distribución de valores de permeabilidad indica que los rangos más típicos de esta propiedad son menores a $148 \mathrm{mD}$ para la arenisca U con un $46 \%$; mientras que el $52 \%$ de resultados de la arenisca $\mathrm{T}$ muestran permeabilidades menores a $236 \mathrm{mD}$.

\subsection{Permeabilidades de probadores de formación con cable}

\subsubsection{Distribución de permeabilidades de las arenas $U$ y $T$}

Las Figuras 10 y 11 muestran los histogramas con la distribución de permeabilidad obtenidos de las areniscas U y $\mathrm{T}$ inferior del campo Shushufindi.

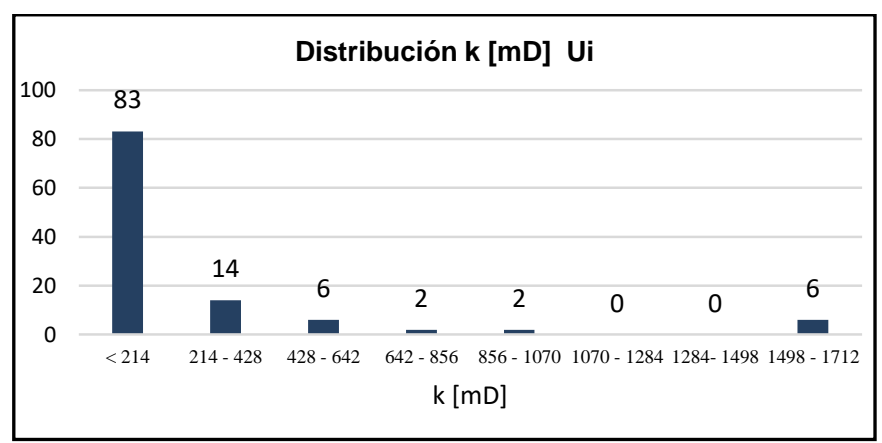

Figura 10. Distribución de permeabilidad efectiva para la arenisca U inferior

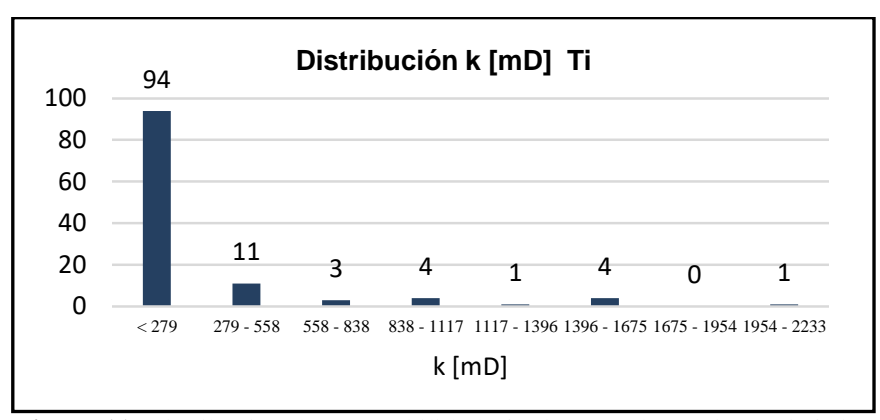

Figura 11. Distribución de permeabilidad efectiva para la arenisca T inferior

La distribución de permeabilidad de estos gráficos permite establecer valores de permeabilidad mayoritariamente distribuida en rangos menores a $214 \mathrm{mD}$ y $279 \mathrm{mD}$ para las areniscas $\mathrm{U}$ y $\mathrm{T}$ respectivamente.

\subsubsection{Comparación permeabilidades de registros y probadores de formación con cable}

La Figura 12 compara las permeabilidades obtenidas con los registros eléctricos y las obtenidas con probadores de formación con cable. 


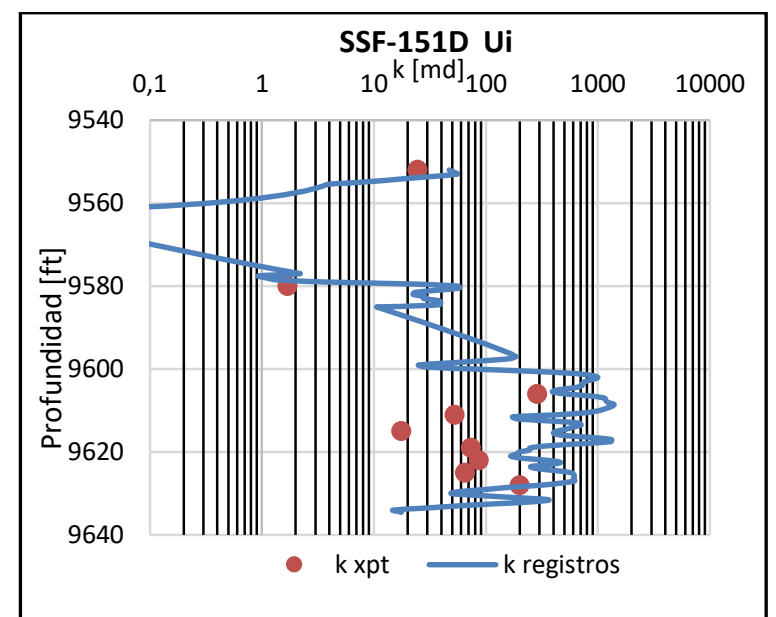

Figura 12. Comparación permeabilidades arenisca U inferior de registros y probadores de formación

La permeabilidad de registros eléctricos es mayor a la obtenida con los probadores de formación, esta diferencia de permeabilidades es común esperarse a pesar que estas dos fuentes se ven afectadas por efecto del filtrado de lodo; el volumen de investigación de estos dos métodos varía; otro factor que ocasiona estas diferencias consiste en el tipo de permeabilidad que determinan, donde la primera de ellas corresponde a una permeabilidad absoluta, mientras la otra es una permeabilidad efectiva.

\subsection{Permeabilidades de análisis transiente de producción}

La Figura 13 compara datos de permeabilidad obtenidos de pruebas de pozos (PTA) y análisis transiente de producción (RTA) para las areniscas $\mathrm{U}$ y $\mathrm{T}$ inferior del campo Shushufindi. La región amarilla corresponde al lugar donde las permeabilidades calculadas a partir de pruebas de pozos son mayores a las determinadas con análisis transiente de producción.

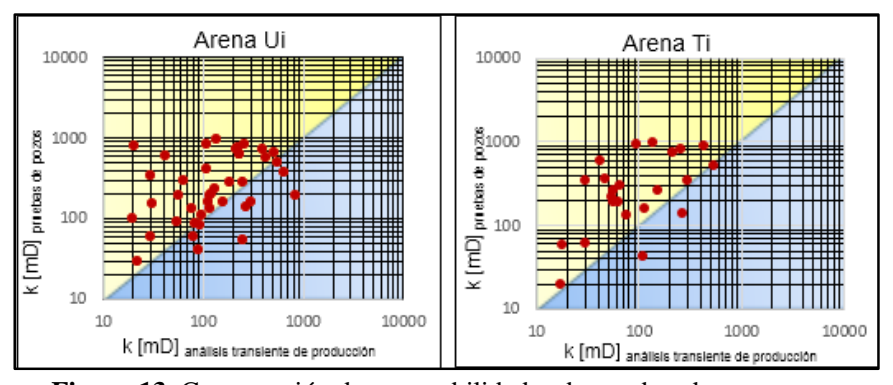

Figura 13. Comparación de permeabilidades de pruebas de pozos con análisis transiente de producción

El $76 \%$ y $87 \%$ de los datos de pruebas de pozos son mayores a los datos RTA, para las areniscas $\mathrm{U}$ y $\mathrm{T}$ inferior respectivamente. Esta diferencia de medidas es de esperarse debido a sus volúmenes de investigación, ya que el RTA tiene como volumen de análisis el área de drenaje del pozo, mientras que las pruebas de pozos dan como resultado un promedio entre el valor máximo y mínimo de permeabilidad medido.

\subsection{Análisis de sensibilidad \\ 3.6.1 Curvas de índice de productividad}

La Figura 14 presenta las curvas de índice productividad (IPR) del pozoSSF-151, estos índices de productividad se calcularon con los valores de permeabilidades obtenidos con cada fuente estudiada, adicionalmente se observa datos de pruebas de producción (Q real).

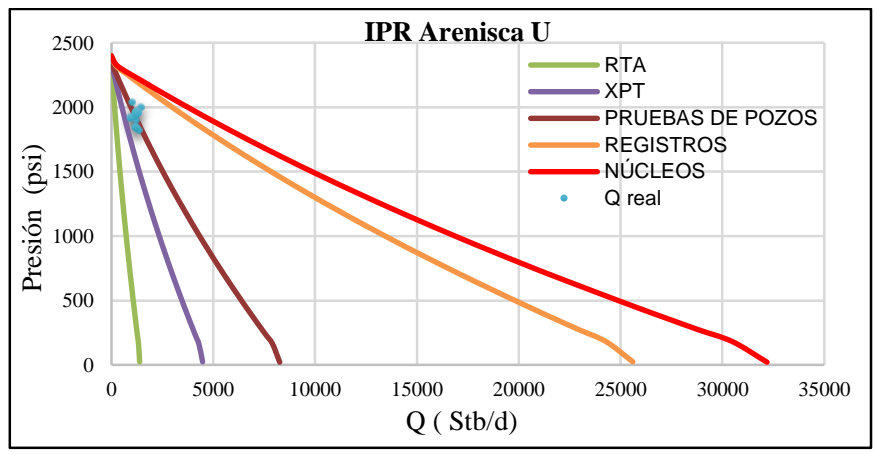

Figura 14. Curvas IPR de la arenisca U pozo SSF-151

Las diferencias en las curvas IPR de cada método son notables, siendo las curvas determinadas con núcleos y registros las de más alto IP, justificado por tratarse de permeabilidades absolutas. El mejor ajuste de las pruebas de producción se observa con la curva IPR generada a partir de la permeabilidad de pruebas de pozos.

La Tabla 3 muestra lo sensible que puede llegar a ser la estimación de la productividad de un pozo según la permeabilidad obtenida con cada método.

Tabla 3. Análisis de sensibilidad arenisca U del pozo SSF-151

\begin{tabular}{lllcc}
\hline \multicolumn{1}{c}{ Q prueba =1208 BFPD Pwf $=\mathbf{1 8 5 0}$ psi } & & & & \\
\cline { 1 - 2 } \multicolumn{1}{c}{ Método } & $\mathrm{K}_{\mathrm{a}}$ & $\mathrm{K}_{\mathrm{e}}$ & $\mathrm{K}[\mathrm{mD}]$ & $\mathrm{Q}[\mathrm{BFPD}]$ \\
\hline Núcleos & $\mathrm{X}$ & & 697 & 5040 \\
Registros & $\mathrm{X}$ & & 554 & 3880 \\
Pruebas de pozos & & $\mathrm{X}$ & 179 & 1250 \\
Probadores de formación con cable & & $\mathrm{X}$ & 97 & 690 \\
Análisis transiente de producción & & $\mathrm{X}$ & 30 & 205 \\
\hline
\end{tabular}

\subsection{Variograma}

En la Figura 15 se observa el variograma de permeabilidades obtenidas de pruebas de presión para diferentes direcciones con su respectiva distancia máxima (h max) de tolerancia para la interpolación de la permeabilidad de la arenisca U del campo Shushufindi.

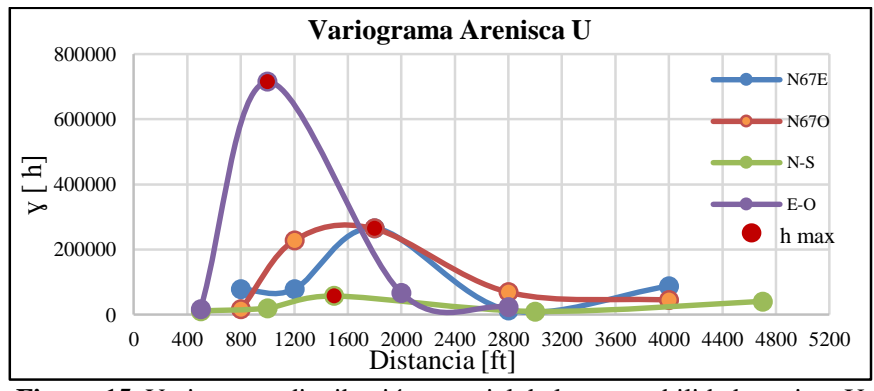

Figura 15. Variograma distribución espacial de la permeabilidad arenisca U del campo Shushufindi 
La evaluación de la populación de datos es una pieza fundamental en la caracterización del reservorio; estableciendo las direcciones y distancias máximas evaluadas para las cuales existe una buena correlación entre los datos de permeabilidad de los pozos de la arenisca U. Estableciéndose una distancia máxima de correlación de 1800 pies para las direcciones $\mathrm{N} 67^{\circ} \mathrm{E}$ y $\mathrm{N} 67^{\circ} \mathrm{O}$; mientras para las direcciones $\mathrm{N}$ $\mathrm{S}$ y E-O las distancias máximas son 1500 y 1000 pies respectivamente.

\section{CONCLUSIONES}

La corrección por efecto de Klinkenberg se vuelve necesaria en núcleos donde se determina bajas permeabilidades (menores a $100 \mathrm{mD}$ ); mientras para valores mayores a los 100 [mD] los valores de permeabilidad medios al aire resultan confiables.

La permeabilidad medida de núcleos es virtualmente la medida más representativa del reservorio a pesar de tratarse de una parte muy pequeña del mismo; constituye una pieza fundamental en la evaluación de registros eléctricos como una fuente de calibración para la selección adecuada de una correlación predictora de la permeabilidad.

Los histogramas muestran una distribución de permeabilidad entre $133-214 \mathrm{mD}$ para la arena $\mathrm{U}$ inferior, mientras que la arena $\mathrm{T}$ inferior esta distribución se halla entre 100-279 mD que corresponden a los rangos de permeabilidad más comunes a encontrar en el campo Shushufindi.

Los datos de permeabilidad obtenidos con los probadores de formación con cable son medidas puntuales y no brindan una medida continua como lo pueden hacer los registros eléctricos.

Se identificó la estrecha relación que existe en los análisis de presión y producción transitoria, ya que comparten fundamentos teóricos muy similares, pero al estimar la permeabilidad se puede apreciar grandes diferencias principalmente por sus diferentes volúmenes de investigación.

Para el Campo Shushufindi el método más apropiado para la estimación de la permeabilidad es a partir de las pruebas de restauración de presión tal como se puede apreciar en el empate de los datos de producción reales con la curva IPR a partir de la permeabilidad generada con esta fuente.

La permeabilidad es un dato que se toma durante diferentes etapas de la vida productiva de un pozo, sin embargo tres métodos son aplicados previo a la vida productiva del pozo: núcleos, registros y los probadores de formación con cable que son tomados durante la etapa de perforación de un pozo, mientras que las pruebas de pozos y el análisis transiente de producción se realiza durante la vida productiva del pozo.

La distribución espacial de la permeabilidad es un aspecto fundamental en la identificacion de heterogeneidades dentro del reservorio.

Todos los métodos en el cálculo de la permeabilidad son de gran importancia en diferentes etapas del gerenciamiento del campo con diferentes funciones ya sea como un dato de calibración, un indicador de evaluación petrofísica o una fuente adecuada de estimación de la productividad de un pozo.

\section{REFERENCIAS}

Al-Harbi, A., Gringarten, A., \& Akkurt, R. (2007). A Practical Approach to Determine Permeability from Wireline Measurements. Society of Petroleum Engineers. doi:10.2118/111221-MS.

Baby, P., Rivadeneira, M., \& Barragán, R. (2014). La Cuenca Oriente: Geología y Petróleo. Quito, Ecuador.

Bazara, M., \& Salman, S. (2009). Permeability Stimation Using Log Data, Abu Dhabi Ofshore Field, UAE. Society of Petroleum Engineers. doi:10.2118/118741-MS.

Cantini, S., Baldini, D., Beretta, E., Loi , D., \& Mazzoni, S. (2013). Reservoir Permeability from Wireline Formation Testers. Society of Petroleum Engineers. doi:10.2118/164924-MS

Castillo, A. O., y Ortega, H. A. (2016). Propiedades Petrofísicas: Análisis, Interpretación y Aplicación en un Pozo Petrolero al Noreste de la República Mexicana. (Tesis pregrado). Universidad Nacional Autónoma de México.

Emery, X. (2013). Geoestadística. Chile: Universidad de Chile

Fanchi, J. (2018). Principles of Applied Reservoir Simulation. Cambridge: Elsevier.

López, F., Biedma, D., \& Suter, A. (2015). Optimizing the Reservoir Management of a Mature Oil Field: The Challenge of Shushufindi. Society of Petroleum Engineers. doi:10.2118/177197-MS.

Mohaghegh, S., Balan, B., \& Armeri, S. (1997). Permeability Determination From Well Log Data. Society of Petroleum Engineers. doi:10.2118/30978-PA.

Nind, T. (2010). Fundamentos de Producción y Mantenimiento de Pozos Petroleros. Limusa.

Pérez, W. (2012). Evaluación del Cálculo de la Movilidad Obtenida de Probadores de Formación con Wireline Usando Información de Registros Convencionales. (Tesis pregrado) Bucaramanga, Colombia: Universidad Industrial de Santander.

Schlumbeger. (2002). Mejoramiento de Nuestra Comprensión acerca de la permeabilidad. Oilfield Review.

Schlumberger. (1996). Wireline Formation Testing and Sampling. Schlumberger Wireline \& Testing.

Schlumberger. (2019). Oilfield Glossary. Obtenido de https://www.glossary.oilfield.slb.com/es/Terms/d/darcy.aspx

Shokir, E., Alsughayer, A., \& Al-Ateeq, A. (2006). Permeability Estimation From Well Log Responses. Petroleum Society of Canada. doi:10.2118/06-11-05

Sullivan, T., Beliveau, D., Kumar, P., \& Tandon, R. (2008). Mangala Field Permeability Measurements: Comparison of Core, 
Wireline, and Well Test Data. Society of Petroleum Engineers. doi:10.2118/113164-MS

Sun, H. (2015). Advanced Production Decline Analysis and Application. ELSEVIER.

Talabani, S., Thamir, F., \& Islam, M. (1999). Rock Permeability Measurements Using Drilling Cutting. Petroleum Society of Canada. doi:10.2118/99-127.

Torbello Ramírez, O. P. (2012). Predicción de Permeabilidad en Yacimientos Altamente Heterogéneos Aplicando Métodos no Convencionales. Quito: Universidad Simón Bolivar.

\section{BIOGRAFÍAS}

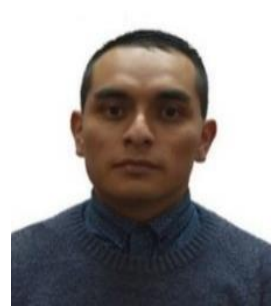

Guamialamá Carlos. Ingeniero de Petróleos de la Facultad de Ingeniería en Geología y Petróleos de la Escuela Politécnica Nacional Quito-Ecuador. Nació en Santo Domingo-Ecuador el 6 de abril de 1994, se graduó de Bachiller en Ciencias Generales en el Instituto Técnico de Comercio Amazonas Santo

Domingo-Ecuador.

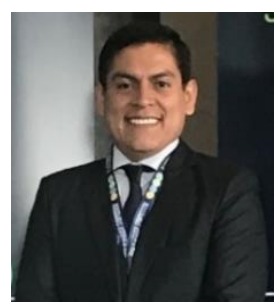

Jose Luis Freire: Ingeniero de Petróleos Escuela Politécnica Nacional (2009). Posee un MSc. Ingeniería en Petróleos mención Optimización de Producción por la Universidad Heriot-Watt UK Scotland graduado con distinción al mérito académico (2014), 10 años en la industria Nacional en PetroAmazonas E.P. como Ingeniero Senior de Operaciones y Completación, e Internacional en Colombia, Argentina, UK, en Schlumberger Production Management como Ingeniero Senior de Reservorios y Producción.

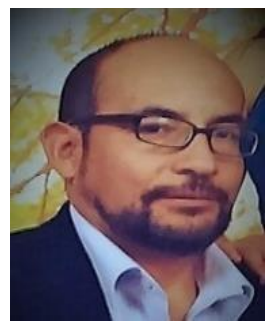

Raúl Valencia. Ingeniero en Petróleos y Magister en Ingeniería Industrial por la Escuela Politécnica Nacional. Profesor Titular a Tiempo completo en la Carrera de Ingeniería en Petróleos desde 1998. Actualmente es jefe del Departamento de Petróleo. Sus intereses de investigación se centran al Área de Petróleos específicamente en la Línea de Yacimientos de Hidrocarburos y Recuperación Mejorada. 